\title{
Postmarketing Surveillance Study of the Efficacy and Safety of Phentermine in Patients with Obesity
}

\author{
Hyun Ok Kim, Jung Ah Lee, Hee Won Suh, Young Sik Kim*, Bum Soo Kim \\ Eun Sook Ahn', Young Jun Roh', Seong Gil Jung ${ }^{4}$, Jin Mok Kim5, \\ Moon Kuk Kang ${ }^{6}$, In Soon Ahn ${ }^{7}$ Young Gyu Park ${ }^{8}$
}

Department of Family Medicine, Asan Medical Center, University of Ulsan College of Medicine, Seoul; ${ }^{1}$ Haemalgeun Clinic, Incheon; ${ }^{2}$ Sungmo Family Medical Clinic, Uijeongbu; ${ }^{3}$ Purun Medical Clinic, Gangneung; ${ }^{4}$ Sejong Clinic, Geoje; ${ }^{5}$ Paradise Clinic, Busan; ${ }^{6}$ Chungdamwhite Clinic, Jinju; ${ }^{7}$ Yonsei Pediatric Clinic, Incheon; ${ }^{8}$ Daejin Medical Center, Seongnam, Korea

Background: Obesity is a complex problem that is now considered a chronic metabolic disease. In Korea, phentermine has been widely used for the treatment of obesity in the primary care setting since 2004 . However, there have been very few studies on the safety and efficacy of phentermine. To investigate the safety and efficacy of this drug, a postmarketing surveillance study was performed.

Methods: A total of 795 patients with obesity (body mass index $\geq 25 \mathrm{~kg} / \mathrm{m}^{2}$ ) were enrolled from 30 primary care centers in Korea from September 2006 to November 2007. Patients were examined to ascertain safety and efficacy at 4-, 8-, and 12week intervals. The criterion for efficacy was defined as a weight loss $\geq 5 \%$ of body weight.

Results: Of the 795 enrolled patients, 735 (92.5\%) were evaluated in safety assessments and 711 (89.4\%) was included in efficacy assessments. A total of 266 adverse events (AEs) were reported by 218 patients (30.6\%), and no serious AEs were reported. Among 711 patients, 324 patients (45.6\%) lost $\geq 5 \%$ of their body weight. The mean weight loss was $3.8 \pm 4.0 \mathrm{~kg}$.

Conclusion: AEs are commonly associated with phentermine, even though phentermine is effective for weight loss and relatively well-tolerated.

Keywords: Phentermine; Obesity; Safety; Efficacy

\section{INTRODUCTION}

Received: August 31, 2011, Accepted: August 22, 2013

${ }^{*}$ Corresponding Author: Young Sik Kim

Tel: +82-2-3010-3811, Fax: +82-2-3010-3815

E-mail: youngkim@amc.seoul.kr

This study is part of a reexamination of phentermine, which was supported by a grant from Huons Co., Ltd (Korea).

Korean Journal of Family Medicine

Copyright (C) 2013 The Korean Academy of Family Medicine

() This is an open-access article distributed under the terms of the Creative Commons Attribution Non-Commercial License (http://creativecommons.org/licenses/by-nc/3.0) which permits unrestricted noncommercial use, distribution, and reproduction in any medium, provided the original work is properly cited.
Obesity is a chronic condition that results from energy imbalance, whereby the patient's energy intake exceeds their total expenditure of energy, resulting in weight gain. ${ }^{1)}$ Obesity is associated with an increased risk of developing chronic illnesses, including hypertension, type II diabetes mellitus, and hyperlipidemia. ${ }^{2)}$ Obesity is defined as a body mass index (BMI) of $\geq 25 \mathrm{~kg} / \mathrm{m}^{2}$ using the Asian-Pacific guidelines. ${ }^{3)}$ Due to the worldwide, rapid increase in obesity, $32 \%$ of the Korean population is considered obese with a $\mathrm{BMI} \geq 25$. $^{4)}$ Therefore, obesity has become a serious public health problem that carries important 
healthcare-related consequences, including increased costs.

Treatments for obesity typically include diet modification, exercise, medication, and cognitive behavioral therapy. Because people typically become obese due to overconsumption that results from an individual's sensitivity to food stimuli and the pleasure derived from eating, appetite suppressants can be used to control eating behavior, food intake, and, thereby, body weight. ${ }^{5)}$ Phentermine is one such appetite regulator. Phentermine is an adrenergic reuptake inhibitor that stimulates the peripheral tissues to secrete noradrenalin. Increased noradrenaline stimulates the $\beta$-adrenergic receptors in the hypothalamus to refrain from food intake and activates the sympathetic nervous system to increase resting energy expenditure. The consequence is weight loss. In addition, this medication increases the effects of serotonin (5-hydroxytryptamine, 5-HT) by suppressing monoamine oxidase and preventing 5-HT from being removed by the lungs. Compared to amphetamine, which has a similar chemical structure to phentermine, phentermine does not affect the secretion and resorption of dopamine, so it has been reported that phentermine has a very low chance of substance abuse or misuse. $^{5-8)}$

Phentermine was approved in 1959. In the 1990s, combination therapy with fenfluramine was reported as effective for regulating weight loss and metabolism, and thus many combined presubscriptions have been administered by doctors. However, this therapy subsequently demonstrated serious side effects, such as cardiac valve disease, and was pushed out of the market. ${ }^{9,10)}$ Also, the Committee of the European Agency for the Evaluation of Medicinal Products decided to temporarily prohibit the use of central appetite suppressants because they demonstrated more risks than benefits. ${ }^{6,10)}$ However, it was also claimed that the assessment of such high risks also included the side effects of fenfluramine (e.g., pulmonary hypertension) and did not exclude the benefits of phentermine. Therefore, it was suggested that research on separate medications was necessary for comparison with long-term research data. It has been reported that the exclusive use of phentermine hardly raises the risk of valvular heart disease or primary pulmonary hypertension. ${ }^{11)}$ There is little data from large-scale studies on the long-term efficacy or safety of phentermine, especially when used as monotherapy. Moreover, there are very few reports on the safety and efficacy of phentermine available on Korean populations. Accordingly, we performed postmarketing surveillance (PMS) research on obese patients who visited hospitals for primary care in order to assess the safety and efficacy of phentermine.

\section{METHODS}

\section{Patients}

A centrally registered PMS system was used to collate data on 837 obese patients who visited 30 primary care physicians in Korea from September 2006 through November 2007. This study was approved of by the research and ethics committee of Asan medical center (study no. 2006-0343). Patients with a $\mathrm{BMI} \geq 25 \mathrm{~kg} / \mathrm{m}^{2}$ (which is used as the cutoff value for obesity in the Asia-Pacific region) ${ }^{3)}$ and who had not been administered phentermine were included in this study. Patients who had uncontrolled hypertension (blood pressure $\geq 140 / 90 \mathrm{~mm} \mathrm{Hg}$ with medication), diagnosed with glaucoma, or were previously treated due to psychological disorders were excluded. Also patients who were receiving drugs that would affect body weight (e.g., topiramate or selective serotonin reuptake inhibitor) or monoamine oxidase inhibitors were excluded. We also excluded patients with a past history of malignancy and patients who had undergone a bariatric operation.

\section{Follow-up Assessments}

Patients were registered at the coordinating center within a week after the first prescription for phentermine and received follow-up examinations every 4, 8, and 12 weeks. The dose of phentermine $(37.5 \mathrm{mg} / \mathrm{d})$ and the prescription bottle were entrusted to their doctors in order to make the experimental conditions as uniform as possible.

\section{Data Collection}

At the time of the first prescription, the patients received a self-administered questionnaire to complete. The questionnaire consisted of items designed to collect data on demographics (e.g., marital status, level of education, monthly income, etc.) and health factors (e.g., obesity medications, smoking and drinking habits, physical activity level, etc.). Regarding smoking history, there were 3 divisions: current, past, or non-smoker. Regarding drinking habits, respondents were asked if they were drinker or 
non-drinker. The questionnaire asked the patients to classify their physical activity level. Physical activity was classified as the frequency of exercise: high (three or more than week), moderate physical activity (1-2 times per week), and low (less than once a week).

\section{Safety and Efficacy Assessments}

The safety assessment was performed using data collected from patients whom the investigators evaluated at least once during the study period. After 12 weeks of follow-up examinations, the compliance rate was measured by the percentage of patients who took medication during the observation period. If the rate was $>80 \%$, compliance was considered "good," and if compliance was $<80 \%$ it was considered "poor." The efficacy test was implemented using patients who received physical measurements and qualified for the safety assessment. The efficacy assessment was performed using data collected from patient who visited the clinic at least once and checked body weight during the study period. The medical treatment was considered effective if the patients lost $>5 \%$ of their body weight. Whenever the patients visited the hospitals, they were checked for weight, waist circumference, blood pressure, and medication status, as well as number of adverse events (AEs) and the reasons for the discontinuation of medication for each patient using the case report form.

The criteria for abdominal obesity included a waist circumference $\geq 90 \mathrm{~cm}$ in men and $\geq 85 \mathrm{~cm}$ in women. To keep all other treatments except medication standardized, physicians and nurses used the guidelines prepared in advance by the research team to inform the patients of the nutrition and exercise guidelines they had to follow.

\section{Statistical Analysis}

Height, body weight, and waist circumference were measured and BMI was calculated as: (weight in kilograms)/(height in meters) $)^{2}$. A paired t-test was used to determine the change of weight and waist circumferences of the subjects. Univariate logistic regression models were used to estimate the odds ratios and 95\% confidence intervals for factors associated with poor efficacy. Also, multivariate logistic regression analysis was performed after adjusting for potential confounding variables including age, sex, smoking status, BMI, physical activity, and compliance. PC-SAS ver. 9.1 (SAS Institute Inc., Cary, NC, USA) was used to perform the statistical analyses and $\mathrm{P}<0.05$ was accepted as significant.

\section{RESULTS}

\section{Baseline Characteristics of the Study Participants}

In total, 795 case report forms were collected and used to analyze data from 735 patients for safety and 711 patients for efficacy (Figure 1). The baseline characteristics of the 795 patients are outlined in Table 1. Most patients were women and the mean age for the whole group was $35.6 \pm 11.1$ years, the mean bodyweight was $94.2 \pm 18.3 \mathrm{~kg}$ for men and $67.8 \pm 11.6$ $\mathrm{kg}$ for women, and the mean BMI was $31.5 \pm 6.0 \mathrm{~kg} / \mathrm{m}^{2}$ for men and $26.8 \pm 4.2 \mathrm{~kg} / \mathrm{m}^{2}$ for women. In total, 458 patients (57.6\%) had a history of receiving anti-obesity medications, 210 patients (26.4\%) were current smokers, and 451 patients (56.7\%) were alcohol drinkers. According to the protocol specified definitions, 44 patients (5.5\%) had controlled hypertension, 23 patients (4.2\%) had hyperlipidemia, and 21 patients $(2.6 \%)$ had type 2 diabetes.

\section{Efficacy of Phentermine}

A total of 711 patients were included in the efficacy analysis. Among them, 441 patients (62.0\%) completed the 12-week treatment protocol and 270 patients $(38.0 \%)$ discontinued the use of phentermine. Weight reduction of $\geq 5 \%$ from baseline was achieved in 324 patients (45.6\%). The changes in anthropometry and blood pressure after 12 weeks of follow-up visits are shown in Table 2. The mean weight reduction was $3.8 \pm 4.0 \mathrm{~kg}(5.2 \% \pm$ $5.0 \%)$, mean BMI reduction was $1.5 \pm 1.5 \mathrm{~kg} / \mathrm{m}^{2}(5.4 \% \pm 5.2 \%)$, and mean change in blood pressure was $2.6 \pm 8.1 \mathrm{~mm} \mathrm{Hg}(1.9 \%$

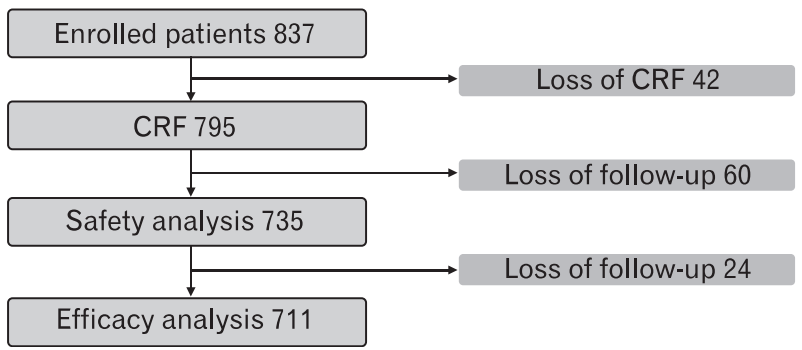

Figure 1. Flow of patients through the trial. CRF: case report form. 
Table 1. Baseline characteristics of study patients $(n=795)$

\begin{tabular}{|c|c|c|}
\hline \multicolumn{2}{|l|}{ Characteristic } & \multirow{2}{*}{$\begin{array}{l}\text { Value } \\
43(5.4)\end{array}$} \\
\hline Sex & Male & \\
\hline & Female & $752(94.6)$ \\
\hline \multirow[t]{5}{*}{ Age (y) } & $<30$ & $288(36.2)$ \\
\hline & $30-39$ & $264(33.2)$ \\
\hline & $40-49$ & $160(20.1)$ \\
\hline & $\geq 50$ & $83(10.5)$ \\
\hline & Mean \pm SD & $35.6 \pm 11.1$ \\
\hline \multirow[t]{3}{*}{ Education (y) } & $<12$ & $54(6.8)$ \\
\hline & 12 & $362(45.5)$ \\
\hline & $>12$ & $261(32.8)$ \\
\hline \multirow[t]{2}{*}{ Marital status } & Married & $390(49.1)$ \\
\hline & Single/divorced & $347(43.7)$ \\
\hline \multirow[t]{3}{*}{ Income (millions of won) } & $<200$ & $126(15.8)$ \\
\hline & 200-399 & $283(35.6)$ \\
\hline & $\geq 400$ & $150(18.9)$ \\
\hline \multirow[t]{3}{*}{ Smoking status } & Nonsmoker & $517(65.0)$ \\
\hline & Ex-smoker & $50(6.3)$ \\
\hline & Smoker & $210(26.4)$ \\
\hline \multirow[t]{2}{*}{ Alcohol consumption } & Nondrinker & 307 (38.6) \\
\hline & Drinker & $451(56.7)$ \\
\hline \multirow[t]{3}{*}{ Physical activity } & Low & $467(58.7)$ \\
\hline & Moderate & $186(23.4)$ \\
\hline & High & $61(7.7)$ \\
\hline \multirow[t]{5}{*}{ Initial dose of phentermine (tablet/d) } & 0.5 & $584(73.5)$ \\
\hline & 0.75 & $8(1.0)$ \\
\hline & 1 & $194(24.4)$ \\
\hline & 1.25 & $1(0.1)$ \\
\hline & 1.5 & $2(0.1)$ \\
\hline \multirow[t]{2}{*}{ Concomitant antiobesity medication } & No & $102(12.8)$ \\
\hline & Yes & $675(84.9)$ \\
\hline \multirow[t]{2}{*}{ Noninvasive procedure } & No & $321(40.4)$ \\
\hline & Yes & $348(43.8)$ \\
\hline \multirow[t]{2}{*}{ Previous antiobesity medication } & No & $458(57.6)$ \\
\hline & Yes & 325 (40.9) \\
\hline
\end{tabular}

Values are presented as number (\%). Missing data were excluded.
Table 2. Changes in anthropometry and blood pressure after 12week treatment with phentermine $(\mathrm{n}=711)$

\begin{tabular}{lc}
\hline \multicolumn{1}{c}{ Variable } & Value \\
\hline Body weight $(\mathrm{kg})$ & $-3.8 \pm 4.0$ \\
Change & $-5.2 \pm 5.0$ \\
\% change & \\
Body mass index $\left(\mathrm{kg} / \mathrm{m}^{2}\right)$ & $-1.5 \pm 1.5$ \\
Change & $-5.4 \pm 5.2$ \\
\% change & \\
Waist circumference $(\mathrm{cm})$ & $-4.1 \pm 4.1$ \\
Change & $-4.5 \pm 4.2$ \\
\% change & \\
Hip circumference $(\mathrm{cm})$ & $-4.0 \pm 6.9$ \\
Change & $-3.8 \pm 6.6$ \\
\% change & \\
Systolic pressure $(\mathrm{mm} \mathrm{Hg})$ & $-1.5 \pm 8.1$ \\
Change & $-1.9 \pm 6.3$ \\
\% change & \\
Diastolic pressure $(\mathrm{mm} \mathrm{Hg})$ & \\
Change & \\
\hline
\end{tabular}

Values are presented as mean $\pm \mathrm{SD}$.

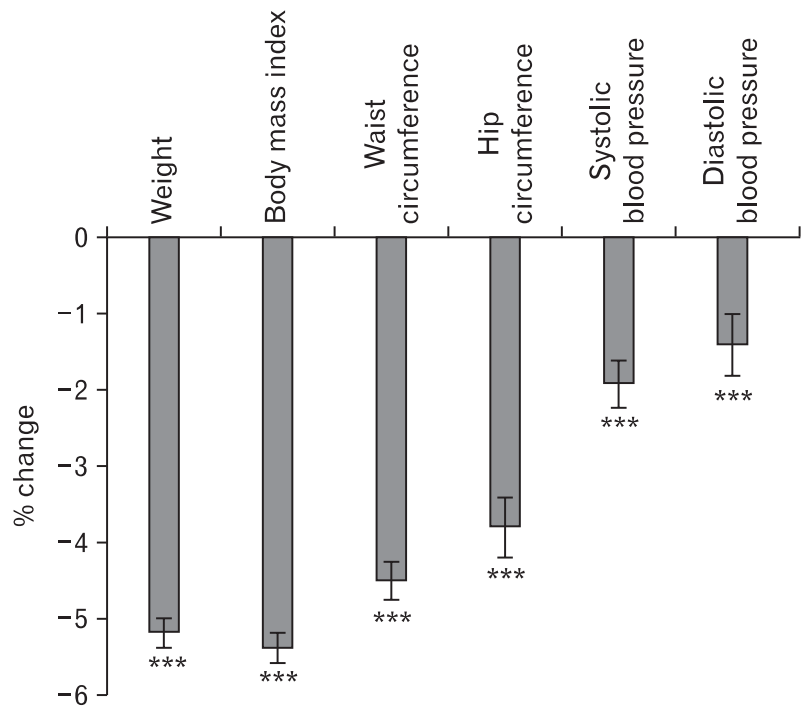

Figure 2. Mean changes in the percentage of anthropometry and blood pressure after 12 weeks of phentermine treatment. ${ }^{* * *} \mathrm{P}<0.001$ for paired t-test. 
Table 3. Factors associated with the poor efficacy of phentermine

\begin{tabular}{|c|c|c|c|c|}
\hline \multirow{2}{*}{ Variable } & \multicolumn{2}{|c|}{ Weight loss (\%) } & \multicolumn{2}{|c|}{ Crude } \\
\hline & $<5$ & $\geq 5$ & Odds ratio & $95 \%$ confidence interval \\
\hline \multicolumn{5}{|l|}{ Sex } \\
\hline Male & $20(5.2)$ & $16(4.9)$ & 1.00 & - \\
\hline Female & 367 (94.8) & $308(95.1)$ & 0.95 & $(0.49-1.87)$ \\
\hline \multicolumn{5}{|l|}{ Age (y) } \\
\hline$<30$ & $129(33.3)$ & $121(37.4)$ & 1.00 & - \\
\hline $30-39$ & $124(32.0)$ & $117(36.1)$ & 0.99 & $(0.70-1.42)$ \\
\hline $40-49$ & $85(22.0)$ & $58(17.9)$ & 1.38 & $(0.91-2.08)$ \\
\hline$\geq 50$ & 49 (12.7) & $28(5.6)$ & 1.64 & $(0.97-2.78)$ \\
\hline \multicolumn{5}{|l|}{ Education (y) } \\
\hline$<12$ & $33(9.7)$ & $16(6.1)$ & 1.00 & - \\
\hline 12 & $176(52.1)$ & $137(52.5)$ & 0.62 & $(0.33-1.18)$ \\
\hline$>12$ & $129(38.2)$ & $108(41.4)$ & 0.58 & $(0.30-1.11)$ \\
\hline \multicolumn{5}{|l|}{ Income (millions of won) } \\
\hline$<200$ & $67(23.8)$ & $45(21.5)$ & 1.00 & - \\
\hline 200-399 & $136(48.4)$ & $109(52.2)$ & 0.84 & $(0.53-1.32)$ \\
\hline$\geq 400$ & $78(27.8)$ & 55 (26.3) & 0.95 & $(0.57-1.59)$ \\
\hline \multicolumn{5}{|l|}{ Marital status } \\
\hline Married & $199(54.8)$ & $154(52.0)$ & 1.00 & - \\
\hline Single/divorced & $164(45.2)$ & $142(48.0)$ & 0.89 & $(0.66-1.22)$ \\
\hline Smoker & $115(30.7)$ & 59 (18.5) & 1.95 & $(1.36-2.79)$ \\
\hline Alcohol drinker & $213(58.7)$ & $181(57.6)$ & 1.04 & $(0.77-1.42)$ \\
\hline \multicolumn{5}{|l|}{ Physical activity } \\
\hline Low & $242(69.3)$ & $169(58.3)$ & 1.00 & - \\
\hline Moderate/high & $107(30.7)$ & $121(41.7)$ & 0.62 & $(0.45-0.86)$ \\
\hline \multicolumn{5}{|l|}{ Body mass index $\left(\mathrm{kg} / \mathrm{m}^{2}\right)$} \\
\hline$<27.0$ & $198(60.6)$ & $131(42.9)$ & 1.00 & - \\
\hline $27.0-29.9$ & $70(21.4)$ & $92(30.2)$ & 0.50 & $(0.34-0.74)$ \\
\hline$\geq 30$ & $59(18.0)$ & $82(26.9)$ & 0.48 & $(0.32-0.71)$ \\
\hline \multicolumn{5}{|l|}{ Compliance (\%) } \\
\hline$<80$ & $358(92.5)$ & $166(51.2)$ & 1.00 & - \\
\hline$\geq 80$ & $29(7.5)$ & $158(48.8)$ & 0.09 & $(0.06-0.13)$ \\
\hline Concomitant antiobesity medication & $330(87.5)$ & $268(84.5)$ & 1.28 & $(0.83-1.98)$ \\
\hline Prior antiobesity medication & $154(40.3)$ & $131(41.2)$ & 0.96 & $(0.71-1.31)$ \\
\hline Noninvasive procedure & $180(57.7)$ & $145(52.0)$ & 1.26 & $(0.91-1.74)$ \\
\hline
\end{tabular}

Values are presented as number (\%). Missing data were excluded. 
$\pm 6.3 \%)$ for systolic pressure and $1.5 \pm 6.7 \mathrm{~mm} \mathrm{Hg}(1.4 \% \pm 8.1 \%)$ for diastolic pressure (Table 2, Figure 2). Factors associated with poor efficacy of phentermine were presented in Table 3. In univariate analysis, smoking status, $\mathrm{BMI}<27 \mathrm{~kg} / \mathrm{m}^{2}$, low physical activity level, and poor compliance were associated with poor efficacy $(P<0.05)$ (Table 3$)$. Multivariate logistic regression analysis was performed for age, sex, and factors associated with poor efficacy in univariate analysis, including smoking status, BMI, physical activity, and compliance (Table 4). Older subjects showed poor efficacy, whereas subjects with good compliance and higher BMI had better efficacy.

\section{Safety of Phentermine}

A total of 266 AEs were reported by 218 patients (30.8\%), the most frequent being insomnia (11.4\%), dry mouth (5.6\%),

Table 4. Multiple logistic regression analysis of factors associated with the poor efficacy of phentermine

\begin{tabular}{|c|c|c|c|}
\hline Variable & Odds ratio & $\begin{array}{l}95 \% \text { confidence } \\
\text { interval }\end{array}$ & P-value \\
\hline Sex & & & 0.415 \\
\hline Male & 1.00 & - & \\
\hline Female & 0.69 & $(0.28-1.69)$ & \\
\hline Age (y) & & & 0.007 \\
\hline$<30$ & 1.00 & - & \\
\hline $30-39$ & 1.33 & $(0.83-2.12)$ & \\
\hline $40-49$ & 2.15 & $(1.23-3.75)$ & \\
\hline$\geq 50$ & 3.19 & $(1.45-7.03)$ & \\
\hline Smoker & 1.40 & $(0.28-2.21)$ & 0.151 \\
\hline Physical activity & & & 0.194 \\
\hline Low & 1.00 & - & \\
\hline Moderate/high & 0.76 & $(0.50-1.15)$ & \\
\hline Body mass index $\left(\mathrm{kg} / \mathrm{m}^{2}\right)$ & & & 0.023 \\
\hline$<27.0$ & 1.00 & - & \\
\hline 27.0-29.9 & 0.63 & $(0.38-1.04)$ & \\
\hline$\geq 30$ & 0.50 & $(0.29-0.86)$ & \\
\hline Compliance (\%) & & & $<0.001$ \\
\hline$<80$ & 1.00 & - & \\
\hline$\geq 80$ & 0.08 & $(0.05-0.13)$ & \\
\hline
\end{tabular}

Missing data were excluded. dizziness $(2.7 \%)$, constipation (2.3\%), nausea (2.3\%), palpitation (1.8\%), headache (1.7\%), anxiety (1.7\%), and tremors $(1.7 \%)$. Sixty-four patients (9.0\%) discontinued phentermine as a direct result of their AEs (Table 5); however, no serious AEs were reported.

\section{DISCUSSION}

The findings of this study show that more than $45 \%$ of the patients who took phentermine lost $\geq 5 \%$ of their body weight, demonstrating a mean weight loss of $3.8 \mathrm{~kg}$, which confirms that the medication is significantly effective for weight loss. On

Table 5. Adverse events reported by the 735 patients administered phentermine

\begin{tabular}{lc}
\hline \multicolumn{1}{c}{ Variable } & Value \\
\hline Insomnia & $84(11.4)$ \\
Dry mouth & $43(5.9)$ \\
Dizziness & $20(2.7)$ \\
Constipation & $17(2.3)$ \\
Nausea & $17(2.3)$ \\
Palpitation & $15(2.0)$ \\
Headache & $12(1.6)$ \\
Anxiety & $12(1.6)$ \\
Tremors & $12(1.6)$ \\
Gastrointestinal disturbance & $5(0.7)$ \\
Overstimulation & $5(0.7)$ \\
Heartburn & $5(0.7)$ \\
Dysphoria & $4(0.5)$ \\
Tachycardia & $3(0.4)$ \\
Weakness & $3(0.4)$ \\
Euphoria & $2(0.3)$ \\
Unpleasant taste & $2(0.3)$ \\
Skin rash & $1(0.1)$ \\
Sweating & $1(0.1)$ \\
Blurred vision & $1(0.1)$ \\
Mental disorder & $1(0.1)$ \\
\hline Flushing & $1(0.1)$ \\
\hline
\end{tabular}

Values are presented as number (\%). 
the other hand, more than $30 \%$ of the patients who took the medication complained of AEs. Although the frequency of AEs was high, only $9.0 \%$ stopped taking the medication and no serious AEs were reported.

In this study, $62.0 \%$ of patients completed the 12 -week phentermine treatment. This completion rate is similar to that reported in an observational study by Kim et al., ${ }^{11)}$ in which the patients showed a $57.4 \%$ completion rate for phendimetrazine, which is also a sympathomimetic drug. In 2005, the Korean Food and Drug Administration (KFDA) recommended short-term (e.g., 4-week) phentermine therapy, which could be followed by an additional 8 weeks following consultation with the patient as long as efficacy was proven. According to this study, only $50 \%$ (or possibly more) of first-time patients wanted additional medication after 4 weeks of treatment, and good efficacy was reported. This shows that the recommendations of the KFDA are appropriate, to some extent.

In our study, $45.6 \%$ of patients lost $\geq 5 \%$ of their total body weight, which was slightly lower than previously reported data (range, 53.9\% to 73.6\%) by Choi et al., ${ }^{12)}$ and Park et al., ${ }^{13)}$ and who assessed the efficacy of phentermine under similar study conditions. However, the mean weight reduction was $3.8 \pm$ $4.0 \mathrm{~kg}(5.2 \% \pm 5.0 \%)$ in our study, and 559 patients $(82.0 \%)$ achieved more than $1.8 \mathrm{~kg}$ weight reduction. Thus, short-term phentermine therapy is considered relatively safe and effective.

There are several problems with prescribing phentermine continuously. The most troublesome aspect is the possibility of abuse or misuse of these kinds of psychotropic appetite suppressants. Although phentermine does not affect the secretion and reuptake of dopamine, negative viewpoints on central appetite suppressants originate from the addictive qualities of amphetamines. Some patients who experienced desirable results demanded that their physicians extend the application of the medication to $>3$ months or repeat the same treatment protocol, which made the physicians uncomfortable. Some patients visit many hospitals in order to receive duplicate prescriptions and take those medications at their discretion. Because these medications are not covered by insurance, the current medical system cannot trace or control such discreet abuse or misuse. Therefore, both the physicians (who prescribe phentermine) and the pharmacists (who compound it) should clearly educate their patients not to abuse or misuse this medication.
AEs of phentermine were very common-about $30 \%$ incidence rate-in this study, but in most cases they were mild. Only $9 \%$ of patients discontinued the use of this medication due to AEs. Therefore, it can be said that, on the whole, patients demonstrated relatively good tolerance of the medication. The most frequent AEs reported in this study included insomnia (11.0\%) and dry mouth (5.6\%). Some domestic studies have reported that dry mouth is the most commonly observed AE, while foreign studies often cite headache as the most common. ${ }^{12,14)}$ Although pulmonary hypertension, ${ }^{15)}$ hypertrophic cardiomyopathy, ${ }^{16)}$ and ischemic stroke have been reported as serious AEs following the administration of amphetaminelike analogues, such as phendimetrazine, these rare AEs were not reported in this study. We expected a rise in blood pressure following the administration of this medication, but no significant difference in blood pressure was noted. This could be explained by the fact that any increase in blood pressure may have been offset by the loss of weight. However, phentermine could still result in habitual intake and trigger withdrawal symptoms such as depression, fatigue, anxiety, tremors, and gastralgia when patients suddenly stop taking the medication.

This study found that good compliance and higher BMI were associated with better efficacy and older age was associated with poor efficacy. These findings are different from other studies, ${ }^{17)}$ one of which reported that patients who demonstrated the best compliance included older patients with chronic diseases and a long history of medications. This discrepancy is believed to originate from the fact that young female patients, and patients with higher social status and income, have a keen interest in obesity treatment and will demonstrate a high level of compliance. It also turned out that combination treatment with other obesity medications reduced body weight. However, it is dangerous to accept these justifications alone because PMS research has its own limitations. Therefore, the efficacy of combination treatments should be compared with those of single medications after the completion of a well-planned clinical trial.

This study is the largest PMS study ever conducted, included almost 800 obese patients, and provides useful data that could be used in the further study of the safety and efficacy of phentermine. Also, our large PMS study is meaningful in the context of the lack of reliable studies done on phentermine. The limitations of this study include the following. First, as we observed the 
participants for only three months, the efficacy of phentermine could be overestimated in our study. Second, this study did not enroll a control group and demonstrates a low efficacy assessment due to some failure in tracing the enrolled patients. However, regarding the assessment of the AEs and the physical measurement of the patients, only those who participated in the educational sessions were allowed to join this research study, in order to minimize evaluation errors and standardize assessments among the researchers. Finally, we cannot exclude the possibility of underestimating the incidence of mild AEs; patients might not have reported AEs due to lack of awareness. However, if those AEs were too minor to report, it would not affect to compliance or discontinuation of phentermine.

In conclusion, we found that phentermine is significantly effective for weight loss. Although patients complained of insomnia and dry mouth, they demonstrated relatively good tolerance to the medication during this period. However, in order to conquer obesity, which is a chronic disease, obesity medications should be evaluated in terms of their safety and efficacy over the long-term. Therefore, better designed and longer clinical research studies will be necessary in the future.

\section{CONFLICT OF INTEREST}

No potential conflict of interest relevant to this article was reported.

\section{REFERENCES}

1. Bray GA. The metabolic syndrome and obesity. Totowa (NJ): Humana Press; 2007.

2. Ioannides-Demos LL, Proietto J, Tonkin AM, McNeil JJ. Safety of drug therapies used for weight loss and treatment of obesity. Drug Saf 2006;29:277-302.

3. World Health Organization; International Association for the Study of Obesity; International Obesity Task Force. The Asia-Pacific perspective: redefining obesity and its treatment. Sydney: Health Communications Australia; 2000.

4. Statistics Korea. Population projections for Korea [Internet]. Daejeon: Statistics Korea; c1996-2009 [cited 2009 Nov 11].
Available from: http://www.kosis.kr/eng/.

5. Kaplan LM. Pharmacological therapies for obesity. Gastroenterol Clin North Am 2005;34:91-104.

6. Halford JC, Boyland EJ, Blundell JE, Kirkham TC, Harrold JA. Pharmacological management of appetite expression in obesity. Nat Rev Endocrinol 2010;6:255-69.

7. Ioannides-Demos LL, Piccenna L, McNeil JJ. Pharmacotherapies for obesity: past, current, and future therapies.J Obes 2011;2011: 179674.

8. Alexander M, Rothman RB, Baumann MH, Endres CJ, Brasic JR, Wong DF. Noradrenergic and dopaminergic effects of (+)-amphetamine-like stimulants in the baboon Papio anubis. Synapse 2005;56:94-9.

9. Cannistra LB, Gaasch WH. Appetite-suppressing drugs and valvular heart disease. Cardiol Rev 1999;7:356-61.

10. Sachdev M, Miller WC, Ryan T, Jollis JG. Effect of fenfluramine-derivative diet pills on cardiac valves: a metaanalysis of observational studies. Am Heart J 2002;144:106573.

11. Kim BS, Kim YS, Ahn ES, Roh YJ, Jung SG, Kim MJ, et al. Postmarketing surveillance study on the efficacy and safety of phendimetrazine in patients with obesity. Korean J Health Promot Dis Prev 2010;10:97-103.

12. Choi CJ, Kim KS, Kim SR, Kang JH, Park HS. Doubleblind, parallel-group, placebo-controlled multi-center clinical trial for evaluating the efficacy and safety of phentermine hydrochloride in obese patients. Korean J Obes 2005; 14:15562.

13. Park HS, Kim KS, Kim BT, Lee KW, Ahn CW, Choi WH. Double-blind, randomized, multi-center, comparative clinical trial of sibutramine mesilate with sibutramine hydrochloride for evaluating efficacy and safety in obese patients. Korean J Obes 2008; 17:82-90.

14. Kim KK, Cho HJ, Kang HC, Youn BB, Lee KR. Effects on weight reduction and safety of short-term phentermine administration in Korean obese people. Yonsei Med J 2006;47:61425.

15. Kay JM. Dietary pulmonary hypertension. Thorax 1994;49 Suppl:S33-8.

16. Rostagno C, Caciolli S, Felici M, Gori F, Neri Serneri GG. Dilated cardiomyopathy associated with chronic consumption of phendimetrazine. Am Heart J 1996;131:407-9.

17. Kim YS, Sunwoo S, Lee HR, Lee KM, Park YW, Shin HC, 
Hyun Ok Kim, et al: Efficacy and Safety of Phentermine

et al. Determinants of non-compliance with lipid-lowering therapy in hyperlipidemic patients. Pharmacoepidemiol Drug Saf 2002; 11:593-600. 